\title{
Spatial behavior of light in second-harmonic generation
}

\author{
Pierre Scotto and Maxi San Miguel \\ Instituto Mediterráneo de Estudios Avanzados, IMEDEA (CSIC-UIB), Campus Universitat Illes Balears, \\ E-07071 Palma de Mallorca, Spain \\ (Received 31 October 2001; published 29 March 2002)
}

\begin{abstract}
We investigate the spatial behavior of nonclassical light produced by type I second-harmonic generation in the traveling-wave configuration. An input-output transformation for the system of fundamental and secondharmonic field is derived in the framework of a linearization approach and used to investigate the properties of an optical system, which consists of a crystal with a quadratic nonlinearity pumped at frequency $\omega$ and enclosed in a two-lens telescopic system. If a faint input image at frequency $2 \omega$ is injected into this device, for a sufficiently large interaction length, the output displays a pair of symmetric amplified versions of the input image at both fundamental and second-harmonic frequency. The analysis of the quantum fluctuations in the output images shows that under certain conditions, this optical device operates noiselessly with respect to the output at frequency $\omega$, whereas the output images at frequency $2 \omega$ are affected by a slight degradation of the signal-to-noise ratio.
\end{abstract}

DOI: 10.1103/PhysRevA.65.043811 PACS number(s): 42.65.Ky, 42.50.Lc, 42.50.Dv, 42.30.-d

\section{INTRODUCTION}

Nonclassical states of light have been the object of intensive studies over the last decade not only because of the interest they represent from a fundamental point of view, but also because they can be used to circumvent standard quantum limits in optical precision measurement and information technologies [1]. A new field of applications of nonclassical light has emerged, since it has been realized that it is possible to produce light with reduced quantum fluctuations not only in time, but also in space, i.e., in the transverse cross section of the beam [2-5]. New developments appear in the field of image processing, with the possibility of noiseless amplification of a faint optical image [6,7] or of image cloning [8], as well as in high-precision optical measurement: here, great precision enhancements with respect to standard methods based on the use of coherent light beams were predicted for the measurement of very small displacements of a light beam $[10,11]$, of very weak spatial phase modulations [12-14], or in the reconstruction of fine details in images in diffractionlimited optical systems [15].

Such light states with a higher spatial order are generated using nonlinear optical media. As a matter of fact, any optical nonlinearity is associated with the simultaneous absorption or emission of photons. On a macroscopic level, and under suitable conditions, this can result in spatial correlations beyond the standard quantum limit, i.e., beyond those corresponding to a random distribution of photons. From this point of view, perhaps the simplest process that is likely to generate nonclassical spatial correlations is the decay of a photon of a given frequency into two photons of lower energy, which is, in principle, possible in any medium presenting a susceptibility with a quadratic nonlinearity. This is precisely the mechanism on which is based the optical parametric amplifier (OPA), a $\chi^{(2)}$-nonlinear crystal illuminated by a strong monochromatic pump field at frequency $2 \omega$. In the crystal, the pump photons may decay either spontaneously or in a stimulated process into a pair of perfectly correlated twin photons. This mechanism gives rise to non- classical quantum correlations between the corresponding spatial frequencies of a parametrically amplified (signal) image and its generated (idler) image, as was demonstrated experimentally in [16]. When enclosed in a classical twolens telescopic system, the OPA is able to generate two amplified copies of a given input image, which are symmetric with respect to the mean light propagation direction, and which are expected to be locally much better correlated than classical copies, not only with respect to intensity fluctuations [8], but also to "phase" fluctuations [9]. Finally, the perfect quantum correlations of the twin photons created in each photon down-conversion process can be used to amplify a faint optical image without deteriorating its signal-tonoise ratio, as was predicted in [7] and realized experimentally in [17].

In this paper, we suggest going one step further along the lines of optical image processing and propose an optical device capable of frequency down-converting a given input image first, and then cloning and amplifying it. If an optical image is injected at frequency $2 \omega$, this device is expected to deliver in the output a pair of amplified versions of this input image, symmetric with respect to the mean light-propagation direction, not only at frequency $\omega$, but also, as a detailed analysis will reveal, at frequency $2 \omega$. In addition, we will show that, under certain conditions, this image processing can be carried out in a way that preserves the signal-to-noise ratio. The realization of these optical operations turns out to be possible using, again, a crystal with a quadratic nonlinearity. But unlike the OPA, for which the pumping is performed at $2 \omega$, we propose here to pump this crystal at the fundamental frequency $\omega$. This new image-processing scheme exploits the well known process of second-harmonic generation (SHG): during propagation through the nonlinear crystal, the fundamental pump field undergoes a conversion into second-harmonic field [18]. The technical advantages of SHG for the generation of squeezed fields have been pointed out in $[19,20]$. Among others, the simplicity of the experimental setup and the possibility to create nonclassical light at both fundamental and second-harmonic frequencies moti- 


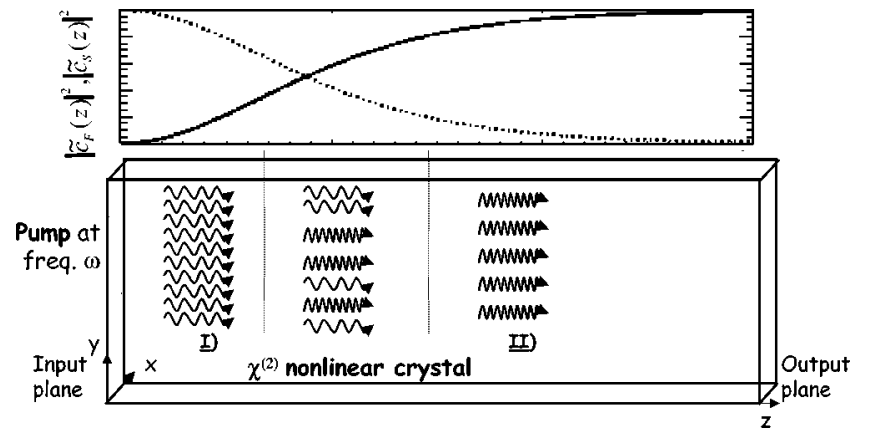

FIG. 1. Second-harmonic generation. Upper part: The intensity of fundamental (dashed line) and second-harmonic field (solid line) inside the crystal as a function of the propagation length in the perfect phase-matched case $\left(2 k_{F}-k_{S}=0\right)$. Lower part: Schematic representation of the different regions of the crystal. The horizontal axis corresponds to the direction of beam propagation, whereas $(x, y)$ defines the transverse plane. The slightly (strongly) wavy arrows represent fundamental (second-harmonic) photons.

vated an extensive investigation work at both experimental and theoretical levels. Now, there are good reasons to believe that the advantages of SHG for squeezing generation could translate into new possibilities for image processing. As a matter of fact, the investigation of the properties of the nonclassical light produced by an OPA showed that squeezing and noiseless amplification are closely related to each other [2]. These aspects, which to our knowledge have not been investigated so far, are relevant in the actual context of the increasing importance of "quantum imaging" for practical applications.

Technically, image-processing problems are solved by investigating the spatial behavior of the fields generated by the optical system under study [2]. Our first objective will be hence to generalize previous investigations devoted to the propagation of quantum fluctuations in SHG [20,21], in order to include the transverse spatial dependence of the fields. This analysis, carried out in Sec. II, allows to derive an input-output transformation for the system of fundamental and second-harmonic fields. In the second part of this paper (Sec. III), we will use these results to investigate SHG-based image processing. First we will focus on the phaseinsensitive configuration, which will give a precise picture of the elementary three-wave processes involved in the formation of the observed output images. We will then concentrate on the phase-sensitive regime and explore the noise properties of this optical device, which plays a central role in quantum imaging.

\section{FIELD-OPERATOR DYNAMICS IN SECOND-HARMONIC GENERATION}

\section{A. General picture}

The interesting possibilities offered by SHG in the field of image processing can be understood from the following simple considerations. Figure 1 shows schematically the physical system we are considering: a $\chi^{(2)}$-nonlinear crystal, which is ideally infinite in the transverse plane, is illuminated by a strong monochromatic pump field at frequency $\omega$.
We restrict ourselves to the simplest case of perfect phase matching. As can be seen from Fig. 1, several regions can be distinguished in the crystal, according to the relative intensities of fundamental and second-harmonic field: ${ }^{1}$ whereas in the part of the crystal close to the input plane, the dominant field will be the fundamental pump field (region I in Fig. 1); an increase of the propagation length leads to a higher conversion of the pump into second-harmonic field through the SHG process, so that at some depth-provided that the nonlinear crystal is long enough-we will reach a region (labeled as region II in Fig. 1) in which the field at frequency $2 \omega$ will be the stronger one. It is easy to show that the regions I and II will show very different behaviors with respect to image processing. To this purpose, we consider the general Hamiltonian describing the three-wave interaction mediated by the quadratic nonlinearity of the crystal,

$$
\begin{aligned}
\hat{H}_{\text {int }}= & i \lambda \sum_{\vec{k}_{1}, \vec{k}_{2}}\left[a_{S}^{\dagger}\left(\vec{k}_{1}+\vec{k}_{2}\right) a_{F}\left(\vec{k}_{1}\right) a_{F}\left(\vec{k}_{2}\right)\right. \\
& \left.-a_{S}\left(\vec{k}_{1}+\vec{k}_{2}\right) a_{F}^{\dagger}\left(\vec{k}_{1}\right) a_{F}^{\dagger}\left(\vec{k}_{2}\right)\right],
\end{aligned}
$$

where $a_{F}(\vec{k})\left[a_{S}(\vec{k})\right]$ annihilates a fundamental (secondharmonic) photon with wave vector $\vec{k}$ and $\lambda$ is the coupling constant of the interaction. The two down-converted photons are considered to have the same polarization, which corresponds to a type-I phase matching. A parametric approximation allows to make meaningful simplifications. In region II, this approximation amounts to replacing the operators associated with the second-harmonic field by the corresponding complex amplitudes. Assuming a homogeneous pumping in the transverse plane, we are left with an effective Hamiltonian

$$
\hat{H}_{i n t, \mathrm{II}}^{e f f}=i \lambda_{\mathrm{II}} \sum_{\vec{k}}\left[a_{F}(\vec{k}) a_{F}(-\vec{k})-a_{F}^{\dagger}(\vec{k}) a_{F}^{\dagger}(-\vec{k})\right],
$$

which coincides with the Hamiltonian of an OPA. As a matter of fact, it describes the creation or annihilation of pairs of twin photons propagating in opposite transverse directions. In region I, the situation is quite different, because now the "strong" field is at frequency $\omega$. In the spirit of the parametric approximation, we can write $a_{F}(\vec{k})$ as $c_{F} \delta(\vec{k})$ $+\left.a_{F}(\vec{k})\right|_{k \neq 0}$, where $c_{F}$ represents the amplitude of the strong homogeneous fundamental pump field. Inserting this expression into Eq. (1) and neglecting the terms that are quadratic in the small quantity $a_{F}(k) / c_{F}$, we obtain, apart from two contributions describing the creation/annihilation of a second-harmonic wave with vanishing wave number, the interaction Hamiltonian

$$
\hat{H}_{i n t, I}^{e f f}=i \lambda_{I} \sum_{\vec{k}}\left[a_{S}^{\dagger}(\vec{k}) a_{F}(\vec{k})-a_{S}(\vec{k}) a_{F}^{\dagger}(\vec{k})\right],
$$

\footnotetext{
${ }^{1}$ The intensities plotted in Fig. 1 as a function of the propagation length are calculated from Eqs. (27) and (28).
} 
which obviously describes a frequency converter: a photon at either frequency $\omega$ or $2 \omega$ with a given wave vector $\vec{k}$ is annihilated and a photon with the same wave vector $\vec{k}$, but at the other frequency, is created. This Hamiltonian is the multimode generalization of that considered in [22] and analyzed in [23].

Combining these two mechanisms gives an idea on how to exploit best SHG for image-processing purposes: An input image at frequency $2 \omega$, superposed to the pump at frequency $\omega$, is expected to first undergo a frequency down-conversion, since it has to cross region I. The result of this first step, i.e., the frequency down-converted version of the input image, arriving then in region II, should be amplified and cloned according to the predictions of the theory of the OPA $[7,8]$. We conclude that injecting an optical image at $2 \omega$, we end up with two symmetric amplified versions of this input at frequency $\omega$.

Of course, this simple argumentation does not take into account, to begin with, the intermediary crystal region, in which both fields generated by the pump inside the crystal have comparable strengths, and hence both mechanisms can occur simultaneously. This makes a full calculation necessary, which will be the object of the remaining of this section. Generalizing the approach developed for the OPA in [2], we will first derive a set of two nonlinearly coupled operator equations, which describe the propagation of the fundamental and second-harmonic field in the nonlinear crystal. They will be solved in the framework of the linearization approach developed in $[21,20]$, based on the assumption that the pumping generates strong monochromatic fields inside the crystal. The main result of this analysis will be an input-output transformation for the system of fundamental and harmonic field, connecting the field operators at the output plane of the crystal with those at the input plane. This transformation will encode the quantum spatial behavior of the light produced by second-harmonic generation.

\section{B. Propagation equations}

The main difference of our analysis with the treatment of the OPA developed in [2] is that in the OPA case, it is generally justified to work in the approximation of a classical undepleted (second-harmonic) pump field, whereas in SHG, pump depletion cannot be neglected and both the fundamental and the second-harmonic field have to be treated simultaneously at a quantum-mechanical level.

First we begin by defining the slowly varying photonannihilation operators for the fundamental and secondharmonic field $\mathcal{A}_{F}(z, \vec{\rho}, t)$ and $\mathcal{A}_{S}(z, \vec{\rho}, t)$ from the positive frequency part of the electric field,

$$
\begin{aligned}
& E_{F}^{(+)}(z, \vec{\rho}, t)=i \xi_{F} \sqrt{\frac{\hbar \omega_{F}}{2 \epsilon_{0} c}} \exp \left[i\left(k_{F} z-\omega_{F} t\right)\right] \mathcal{A}_{F}(z, \vec{\rho}, t), \\
& E_{S}^{(+)}(z, \vec{\rho}, t)=i \xi_{S} \sqrt{\frac{\hbar \omega_{S}}{2 \epsilon_{0} c}} \exp \left[i\left(k_{S} z-\omega_{S} t\right)\right] \mathcal{A}_{S}(z, \vec{\rho}, t) .
\end{aligned}
$$

The wave numbers of fundamental and second-harmonic wave in the nonlinear medium, $k_{F}$ and $k_{S}$, depend on the wave frequency through the dispersion relation $\omega=\omega(k)$. In Eqs. (4) and (5), the prefactors

$$
\xi_{\sigma}=\frac{u\left(k_{\sigma}\right) v\left(k_{\sigma}\right)}{c^{2} \cos \rho\left(k_{\sigma}\right)}
$$

involve the group velocity $u\left(k_{\sigma}\right)$, the phase velocity $v\left(k_{\sigma}\right)$, and some generalized anisotropy angle $\rho\left(k_{\sigma}\right)$. They describe the strength of the electric field in the medium, as compared to that in the vacuum. $z$ is the coordinate on the longitudinal axis, which is defined as the beam axis, and $\vec{\rho}$ is the twodimensional coordinate vector in the transverse plane.

To describe the dynamics of these two fields in a $\chi^{(2)}$ nonlinear crystal, we choose, following [2], a Hamiltonian formulation, based on the following Hamiltonian operator:

$$
\hat{H}=\hat{H}_{0, F}+\hat{H}_{0, S}+\hat{H}_{i n t}
$$

in which $\hat{H}_{0, F}$ and $\hat{H}_{0, S}$ are the free-field Hamiltonians for the fundamental and the second-harmonic field in the medium, whereas $\hat{H}_{\text {int }}$ describes the interaction between the two fields generated by the nonlinearity of the crystal. In terms of the slowly varying operators $\mathcal{A}_{\sigma}(z, \vec{\rho}, t), \sigma=F, S$, the free-field Hamiltonians are given by the expression [2]

$$
\hat{H}_{0, \sigma}=\frac{\hbar \omega_{\sigma}}{c} \int_{V} d z d^{2} \rho \mathcal{A}_{\sigma}^{\dagger}(z, \vec{\rho}, t) \mathcal{A}_{\sigma}(z, \vec{\rho}, t),
$$

in which the space integration is extended to the whole volume of the crystal. The expectation value $\left\langle\mathcal{A}_{\sigma}^{\dagger}(z, \vec{\rho}, t) \mathcal{A}_{\sigma}(z, \vec{\rho}, t)\right\rangle$ can be interpreted as the energy density per unit volume, scaled by a factor $\hbar \omega_{\sigma} / c$. The interaction part $\hat{H}_{i n t}$ describes a three-wave interaction, which, under the usual assumption of a instantaneous and local nonlinear response of the medium [24], is given by

$$
\begin{aligned}
\hat{H}_{i n t}= & \chi^{(2)} \int_{V} d z d^{2} \rho E_{S}^{(+)}(z, \vec{\rho}, t) E_{F}^{(-) 2}(z, \vec{\rho}, t) \\
& +\chi^{(2)} \int_{V} d z d^{2} \rho E_{S}^{(-)}(z, \vec{\rho}, t) E_{F}^{(+) 2}(z, \vec{\rho}, t),
\end{aligned}
$$

and can be rewritten in terms of the slowly varying field operators,

$$
\begin{aligned}
\hat{H}_{i n t}= & i \hbar \lambda\left[\int_{V} d z d^{2} \rho \exp [i \Delta k z] \mathcal{A}_{S}^{\dagger}(z, \vec{\rho}, t) \mathcal{A}_{F}^{2}(z, \vec{\rho}, t)\right. \\
& \left.-\int_{V} d z d^{2} \rho \exp [-i \Delta k z] \mathcal{A}_{S}(z, \vec{\rho}, t) \mathcal{A}_{F}^{\dagger 2}(z, \vec{\rho}, t)\right],
\end{aligned}
$$

with a coupling constant $\hbar \lambda=\chi^{(2)}\left(\hbar / 2 \epsilon_{0} c\right)^{3 / 2} \xi_{F}^{2} \xi_{S} \sqrt{\omega_{F}^{2} \omega_{S}}$ proportional to the susceptibility constant $\chi^{(2)}$ of the medium. $\Delta k=2 k_{F}-k_{S}$ is generally referred to as the collinear 
phase mismatch. $\hat{H}_{i n t}$ is the sum of two contributions that display the two basic and complementary processes, which connect the fundamental and second-harmonic fields in a $\chi^{(2)}$-nonlinear crystal: the first term in Eq. (10) is responsible for second-harmonic generation, whereas the second contribution, which has to be present because of the Hermiticity requirement imposed on $\hat{H}_{i n t}$, will induce the process of photon down-conversion. The dynamics of the two field operators are described by the Heisenberg equations

$$
\partial_{t} \mathcal{A}_{\sigma}(z, \vec{\rho}, t)=i \omega_{\sigma} \mathcal{A}_{\sigma}(z, \vec{\rho}, t)+\frac{i}{\hbar}\left[\hat{H}, \mathcal{A}_{\sigma}(z, \vec{\rho}, t)\right],
$$

which, for the Hamiltonian defined by Eqs. (8) and (10), take the following explicit form:

$$
\begin{aligned}
\partial_{t} \mathcal{A}_{F}(z, \vec{\rho}, t)= & i \omega_{F} \mathcal{A}_{F}(z, \vec{\rho}, t)-i \omega_{F} \int_{V} d z^{\prime} d^{2} \rho^{\prime} \\
& \times G_{F}\left(z-z^{\prime}, \vec{\rho}-\vec{\rho}^{\prime}\right) \mathcal{A}_{F}\left(z^{\prime}, \vec{\rho}^{\prime}, t\right)-2 c \lambda \int_{V} d z^{\prime} \\
& \times d^{2} \rho^{\prime} G_{F}\left(z-z^{\prime}, \vec{\rho}-\vec{\rho}^{\prime}\right) \exp \left(-i \Delta k z^{\prime}\right) \\
& \times \mathcal{A}_{S}\left(z^{\prime}, \vec{\rho}^{\prime}, t\right) \mathcal{A}_{F}^{\dagger}\left(z^{\prime}, \vec{\rho}^{\prime}, t\right), \\
\partial_{t} \mathcal{A}_{S}(z, \vec{\rho}, t)= & i \omega_{S} \mathcal{A}_{S}(z, \vec{\rho}, t)-i \omega_{S} \int_{V} d z^{\prime} d^{2} \rho^{\prime} \\
& \times G_{S}\left(z-z^{\prime}, \vec{\rho}-\vec{\rho}^{\prime}\right) \mathcal{A}_{S}\left(z^{\prime}, \overrightarrow{\rho^{\prime}}, t\right) \\
& +c \lambda \int_{V} d z^{\prime} d^{2} \rho^{\prime} G_{S}\left(z-z^{\prime}, \vec{\rho}-\vec{\rho}^{\prime}\right) \\
& \times \exp \left(i \Delta k z^{\prime}\right) \mathcal{A}_{F}^{2}\left(z^{\prime}, \vec{\rho}^{\prime}, t\right),
\end{aligned}
$$

with

$$
\begin{aligned}
G_{\sigma}\left(z-z^{\prime}, \vec{\rho}-\vec{\rho}^{\prime}\right)= & \int \frac{d k_{z} d^{2} q}{(2 \pi)^{3}} \frac{\omega\left(\sqrt{k_{z}^{2}+\vec{q}^{2}}\right)}{\omega_{\sigma}} \exp \left[i\left(k_{z}-k_{\sigma}\right)\right. \\
& \left.\times\left(z-z^{\prime}\right)+i \vec{q}\left(\vec{\rho}-\vec{\rho}^{\prime}\right)\right] .
\end{aligned}
$$

To make further progress, we first have to derive from Eqs. (12) and (13), more transparent propagation equations. Rather than working in real space, it is convenient to introduce quantum operators associated with waves with a given transverse wave vector $\vec{q}$ and temporal frequency offset $\Omega$ (with respect to the corresponding carrier frequency $\omega_{\sigma}$ ). As in the OPA case, starting from the Fourier transform of the field operators

$$
\mathcal{A}_{\sigma}(z, \vec{q}, \Omega)=\int d^{2} \rho e^{-i \vec{q} \cdot \vec{\rho}} \int d t e^{i \Omega t} \mathcal{A}_{\sigma}(z, \vec{\rho}, t),
$$

it is advantageous to separate the effects of free propagation through the crystal from the effects of the interaction induced by the nonlinearity of the crystal. This is achieved by defining, for each field, a propagation-corrected Fourier amplitude

$$
\hat{A}_{\sigma}(z, \vec{q}, \Omega)=\xi_{\sigma} \sqrt{n_{\sigma}} \exp \left\{-i\left[k_{\sigma}^{z}(\vec{q}, \Omega)-k_{\sigma}\right] z\right\} \mathcal{A}_{\sigma}(z, \vec{q}, \Omega),
$$

where $k_{\sigma}^{z}(\vec{q}, \Omega)=\sqrt{k\left(\omega_{\sigma}+\Omega\right)^{2}-\vec{q}^{2}}$, which involves the wave number $k\left(\omega_{\sigma}+\Omega\right)$ associated with the frequency $\omega_{\sigma}$ $+\Omega$ through the dispersion relation, represents the longitudinal wave number of a wave with frequency $\omega_{\sigma}+\Omega$ and transverse wave vector $\vec{q}$. Clearly, the exponential phase factor in Eq. (16) is chosen so as to absorb, in the freepropagation case, the exact $z$ dependence of the wave with frequencies $\left(\vec{q}, \omega_{\sigma}+\Omega\right)$ associated with the field operator $\hat{A}_{\sigma}(z, \vec{q}, \Omega)$. The additional prefactor $\xi_{\sigma} \sqrt{n_{\sigma}}=\sqrt{u_{\sigma} / c}$, with $u_{\sigma}$ defined as the group velocity of a wave with frequency $\omega_{\sigma}$, allows to identify $\left\langle\hat{A}_{\sigma}^{\dagger}(z, \vec{\rho}, t) \hat{A}_{\sigma}(z, \vec{\rho}, t)\right\rangle$ with the mean photon flux density in the medium in (photons $/ \mathrm{cm}^{2} / \mathrm{sec}$ ). Moreover, it can be shown that $\hat{A}_{F}(z, \vec{q}, \Omega)$ and $\hat{A}_{S}(z, \vec{q}, \Omega)$ fulfill the standard commutation relations

$$
\begin{aligned}
{\left[\hat{A}_{\sigma}(z, \vec{q}, \Omega), \hat{A}_{\sigma^{\prime}}^{\dagger}\left(z, \vec{q}^{\prime}, \Omega^{\prime}\right)\right]=} & (2 \pi)^{3} \delta_{\sigma \sigma^{\prime}} \delta^{(2)}\left(\vec{q}-\vec{q}^{\prime}\right) \\
& \times \delta\left(\Omega-\Omega^{\prime}\right) .
\end{aligned}
$$

In the standard paraxial $\left[|\vec{q}| \ll k_{\sigma}^{z}(\vec{q}, \Omega)\right]$ and quasimonochromatic $\left(\Omega \ll \omega_{\sigma}\right)$ approximation and under the assumption of a slow $z$ dependence of the field operators, it can be shown that the propagation-corrected Fourier amplitudes $\hat{A}_{F}(z, \vec{q}, \Omega)$ and $\hat{A}_{S}(z, \vec{q}, \Omega)$ obey the following set of coupled propagation equations:

$$
\begin{aligned}
\frac{\partial}{\partial z} \hat{A}_{F}(z, \vec{q}, \Omega)= & -2 K \int d^{2} q^{\prime} d \Omega^{\prime} \hat{A}_{F}^{\dagger}\left(z, \vec{q}^{\prime}, \Omega^{\prime}\right) \hat{A}_{S}(z, \vec{q} \\
& \left.+\vec{q}^{\prime}, \Omega+\Omega^{\prime}\right) \exp \left\{i \left[k_{S}^{z}\left(\vec{q}+\vec{q}^{\prime}, \Omega+\Omega^{\prime}\right)\right.\right. \\
& \left.\left.-k_{F}^{z}(\vec{q}, \Omega)-k_{F}^{z}\left(\vec{q}^{\prime}, \Omega^{\prime}\right)\right] z\right\} \\
\frac{\partial}{\partial z} \hat{A}_{S}(z, \vec{q}, \Omega)= & +K \int d^{2} q^{\prime} d \Omega^{\prime} \hat{A}_{F}\left(z, \vec{q}^{\prime}, \Omega^{\prime}\right) \hat{A}_{F}(z, \vec{q} \\
& \left.-\vec{q}^{\prime}, \Omega-\Omega^{\prime}\right) \exp \left\{i \left[k_{F}^{z}\left(\vec{q}^{\prime}, \Omega^{\prime}\right)\right.\right. \\
& \left.\left.+k_{F}^{z}\left(\vec{q}-\vec{q}^{\prime}, \Omega-\Omega^{\prime}\right)-k_{S}^{z}(\vec{q}, \Omega)\right] z\right\},
\end{aligned}
$$

where $K=(2 \pi)^{-3} \sqrt{c^{3} / u_{F}^{2} u_{S}} \lambda$ is the coupling constant of the interaction. These nonlinearly coupled differential-operator equations describe the propagation of fundamental and second-harmonic field through the nonlinear medium. The right-hand side (rhs) of Eqs. (18) and (19) represent a sum over all three wave processes that are able to generate a fundamental and a second-harmonic wave, respectively, with frequencies $(\vec{q}, \Omega)$. The only physical constraints are energy and transverse and longitudinal momentum conservation, which Eqs. (18) and (19) can be shown to fulfill. Equations (18) and (19) generalize the propagation equations derived in [21] without considering the transverse spatial dependence of 
the fields, as well as those considered in [20], in which a single temporal mode for each field was taken into account. Furthermore, specializing Eqs. (18) and (19) for the special case of a strong undepleted classical field at secondharmonic frequency, one retrieves the equation describing the propagation of the fundamental field in an OPA, either in the plane-wave approximation [2] or for a more general pump-field distribution [25].

Being able to solve Eqs. (18) and (19) in the general case, one could express the field operators in the output plane of the crystal as a functional of the field operators in the input plane, which would finally allow to calculate, for any arbitrary quantum-mechanical state of the electromagnetic field illuminating the crystal, the state of the outcoming radiation. Of course, due to the nonlinear nature of these equations, some suitable approximation scheme is needed, as described below.

\section{Two-field input-output relations}

In the context of SHG, the standard linearized-fluctuation analysis is generally justified by considering that the strong incoming pump beam of monochromatic light at frequency $\omega$ generates inside the nonlinear crystal a strong secondharmonic beam at frequency $2 \omega[21,20]$. The strong intensity assumption allows, in principle, to treat the quantum fluctuations of the fields around their mean values as small perturbations, and to linearize Eqs. (18) and (19) with respect to $\hat{A}_{\sigma}(z, \vec{q}, \Omega)-\left\langle\hat{A}_{\sigma}(z, \vec{q}, \Omega)\right\rangle$. In fact, a close inspection of Eqs. (18) and (19) reveals that in the case of SHG, it is enough to require the quantum fluctuations to be much weaker than the field mean amplitude for the fundamental field. This observation justifies the validity of the linearization procedure in the neighborhood of the input plane, in spite of a vanishing second-harmonic amplitude. But this requirement is no longer fulfilled, as can be seen from Fig. 1, in the limit of large propagation lengths, provoking a breakdown of this approach, as will be discussed in some detail below.

Going along the lines of the linearized-fluctuation analysis, we consider here a slightly more general situation, which corresponds to image-processing problems: we assume the field distribution in the input plane of the crystal to be given by the superposition of a strong uniform pump field at frequency $\omega$ and some "weak" coherent signal at frequency $2 \omega$, with an arbitrary space-time distribution representing an input optical image. The propagation of this signal through the nonlinear crystal can be described in the framework of the linearization approach, provided that at any point inside the crystal the fundamental field generated by the input signal remains weak with respect to the depleted pump field.

Following [21] and [20] we hence write the propagationcorrected field operators associated with fundamental and second-harmonic field as

$$
\begin{aligned}
& \hat{A}_{F}(z, \vec{q}, \Omega)=c_{F}(z) \delta^{(2)}(\vec{q}) \delta(\Omega)+\hat{a}_{F}(z, \vec{q}, \Omega), \\
& \hat{A}_{S}(z, \vec{q}, \Omega)=c_{S}(z) \delta^{(2)}(\vec{q}) \delta(\Omega)+\hat{a}_{S}(z, \vec{q}, \Omega),
\end{aligned}
$$

where $c_{F}(z)$ and $c_{S}(z)$ are the amplitudes of the strong monochromatic waves at frequencies $\omega$ and $2 \omega$ generated by the pump inside the crystal, which, for simplification, are considered in the plane-wave approximation, and $\hat{a}_{F}(z, \vec{q}, \Omega)$ and $\hat{a}_{S}(z, \vec{q}, \Omega)$ are the quantum field operators associated with the two fields. These take into account the propagation of any field distribution injected into the crystal in addition to the strong pump field. In particular, they encode the propagation of the vacuum quantum fluctuations entering the crystal through its input plane, which are responsible for the quantum fluctuations in the output fields, as analyzed in $[21,20]$.

Inserting Eqs. (20) and (21) into Eqs. (18) and (19), and keeping only the terms up to the first order in $\hat{a}_{F}(z, \vec{q}, \Omega)$ and $\hat{a}_{S}(z, \vec{q}, \Omega)$, we find, equating the zeroth-order terms, that $c_{F}(z)$ and $c_{S}(z)$ have to fulfill the well-known classical propagation equation of nonlinear optics

$$
\begin{gathered}
\frac{d}{d z} c_{F}(z)=-2 K c_{F}^{*}(z) c_{S}(z) e^{-i \Delta k z}, \\
\frac{d}{d z} c_{S}(z)=+K c_{F}^{2}(z) e^{i \Delta k z}
\end{gathered}
$$

Defining a dimensionless characteristic interaction length

$$
\zeta=\sqrt{2 W} K z,
$$

which is proportional to the square root of the input power $W=\left|c_{F}(0)\right|^{2}+2\left|c_{S}(0)\right|^{2}$ injected into the nonlinear crystal and to the strength of the nonlinearity $K$, Eqs. (22) and (23) can be conveniently rewritten in terms of the following dimensionless field amplitudes $\tilde{c}_{F}(z)=c_{F}(z) / \sqrt{W}$ and $\tilde{c}_{S}(z)$ $=c_{S}(z) / \sqrt{W / 2}$ as

$$
\begin{gathered}
\frac{d}{d \zeta} \tilde{c}_{F}(\zeta)=-\tilde{c}_{F}^{*}(\zeta) \tilde{c}_{S}(\zeta) e^{-i \Delta s \zeta}, \\
\frac{d}{d \zeta} \tilde{c}_{S}(\zeta)=+\tilde{c}_{F}^{2}(\zeta) e^{i \Delta s \zeta}
\end{gathered}
$$

where $\Delta s=\Delta k z_{0}, z_{0}=1 /(\sqrt{2 W} K)$ being the typical length scale involved in Eq. (24), represents the dimensionless collinear phase mismatch. It can be easily checked that the quantity $\left|\tilde{c}_{F}(\zeta)\right|^{2}+\left|\tilde{c}_{S}(\zeta)\right|^{2}=1$ is a constant of motion. This is the well-known Manley-Rowe relation, which expresses the conservation of energy flow in the lossless crystal. The coupled equations (25) and (26) can be solved analytically [18]. In the case of a vanishing phase mismatch $\Delta k=0$, the solution is particularly simple and reads

$$
\begin{aligned}
& \tilde{c}_{F}(\zeta)=e^{i \phi_{F}^{(0)}} \operatorname{sech}(\zeta), \\
& \tilde{c}_{S}(\zeta)=e^{2 i \phi_{F}^{(0)} \tanh (\zeta) .}
\end{aligned}
$$

where $\phi_{F}^{(0)}$ is the phase of the pump field. These expressions correspond to the profiles plotted in Fig. 1. 
Collecting now the terms at first order, we obtain the following equations of propagation for the quantum field operators $\hat{a}_{F}(z, \vec{q}, \Omega)$ and $\hat{a}_{S}(z, \vec{q}, \Omega)$ :

$$
\begin{aligned}
\frac{\partial}{\partial \zeta} \hat{a}_{F}(\zeta, \vec{q}, \Omega)= & -\tilde{c}_{S}(\zeta) \hat{a}_{F}^{\dagger}(\zeta,-\vec{q},-\Omega) e^{-i \widetilde{\Delta}(\vec{q}, \Omega) \zeta} \\
& -\sqrt{2} \tilde{c}_{F}^{*}(\zeta) \hat{a}_{S}(\zeta, \vec{q}, \Omega) e^{-i \widetilde{D}(\vec{q}, \Omega) \zeta}, \\
\frac{\partial}{\partial \zeta} \hat{a}_{S}(\zeta, \vec{q}, \Omega)= & +\sqrt{2} \tilde{c}_{F}(\zeta) \hat{a}_{F}(\zeta, \vec{q}, \Omega) e^{i \widetilde{D}(\vec{q}, \Omega) \zeta},
\end{aligned}
$$

which involve two different dimensionless phase-mismatch functions,

$$
\begin{gathered}
\widetilde{\Delta}(\vec{q}, \Omega)=\left[k_{F}^{z}(\vec{q}, \Omega)+k_{F}^{z}(-\vec{q},-\Omega)-k_{S}\right] z_{0}, \\
\widetilde{D}(\vec{q}, \Omega)=\left[k_{F}^{z}(\vec{q}, \Omega)+k_{F}-k_{S}^{z}(\vec{q}, \Omega)\right] z_{0} .
\end{gathered}
$$

Before analyzing the physical content of these equations, it is important to discuss the range of validity of the linearization approach. As a matter of fact, in the perfect phase-matched case, the linearized-fluctuation analysis predicts that the fundamental field should evolve, in the limit of large interaction lengths, into a perfect amplitude-squeezed vacuum [21]. This leads to a clear contradiction of the basic requirement of the linearization approach that at frequency $\omega$, the amplitude of the fluctuations should be much smaller than the mean value of the field [26]. This observation was the starting point of an extended work in which the predictions of the linearized theory for traveling-wave SHG were compared to the results of the stochastic integration of the full nonlinear propagation equations obtained in the positive $P$ representation. This study was carried out with respect to the field mean values [26], squeezing properties [27], and quantum nondemolition criteria [28], with the following main conclusions: as far as the field mean values or the quadrature squeezing either for each field separately or for their sum and difference are concerned, the linearization approach was found to give very accurate results for interaction lengths $\zeta \leqslant 4$. For the squeezing in intensity, its range of validity slightly shrinks to $\zeta \leqslant 3$. Finally, with respect to a description of the behavior of the fields in terms of standard QND criteria, it was shown that the breakdown of the linearization approach occurs for even smaller interaction lengths $\zeta \sim 2$. For these very sensitive quantities, not even the truncated Wigner representation was found to reproduce the exact results [28,29]. Since our analysis is based on linearization, it is important to keep these results in mind. As will be clear from the remaining of this paper, the quantities we will mainly be interested in are calculated from the squeezing spectra for given field quadratures. We can hence estimate the upper bound of validity of the analysis presented here as $\zeta \sim 4$.

With this restriction, we can now turn to the interpretation of Eqs. (29) and (30). Inspecting first Eq. (29), two sources of fundamental waves with frequencies $(\vec{q}, \Omega)$ can be distinguished. The first source term on the rhs of Eq. (29) reflects process I of Fig. 2: photons of the strong homogeneous second-harmonic wave generated by the pump inside the
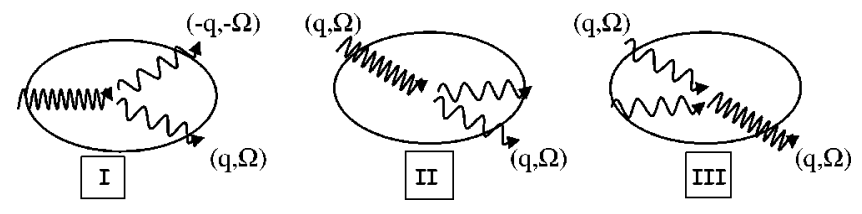

FIG. 2. Different three-wave processes involving one photon of one of the two strong homogeneous fields generated by the pump inside the crystal. The slightly (strongly) wave-shaped arrows represent waves at fundamental (second-harmonic) frequency. Process I represents twin-photon emission, process II (III) is a down- (up-) conversion process at wave number $q$, which occurs under radiation (absorption) of a fundamental pump photon.

crystal may split into two fundamental photons with opposite frequency offsets $\Omega$ and $-\Omega$ and transverse wave vectors $\vec{q}$ and $-\vec{q}$. This process, which is described by the effective Hamiltonian (2), generates a coupling between the amplitudes $\hat{a}_{F}(z, \vec{q}, \Omega)$ and $\hat{a}_{F}^{\dagger}(z,-\vec{q},-\Omega)$. The specificity of the SHG scheme is entirely due to the presence of a strong uniform field at fundamental frequency, which, as already mentioned, dominates in region I of the crystal (see Fig. 1). This strong fundamental field stimulates the process labeled II in Fig. 2, which describes the frequency down-conversion of a second-harmonic wave with $(\vec{q}, \Omega)$ into a fundamental wave with $(\vec{q}, \Omega)$ and hence translates into a coupling between the field operators $\hat{a}_{F}(z, \vec{q}, \Omega)$ and $\hat{a}_{S}(z, \vec{q}, \Omega)$. This is the physical content of the second source term in Eq. (29), which, in terms of a Hamiltonian formulation, can be traced back to the effective Hamiltonian (3). Energy conservation implies that this frequency-changing process occurs under the radiation of a fundamental pump photon. The reversed process (process III in Fig. 2) acts as a source of second-harmonic photons, as displayed by the rhs of Eq. (30).

As can be seen from the structure of the propagation equations (29) and (30), two factors determine the efficiency of a given elementary three-wave process: the amplitude of the strong homogeneous wave involved in that particular process and the phase-mismatch function, which is the effective phase mismatch within the crystal along the beampropagation direction. A large phase mismatch results in fast spatial oscillations of the source term, which reduces the efficiency of this particular process. For this reason, process I will be important provided that $\widetilde{\Delta}(\vec{q}, \Omega) \zeta \ll 1$, whereas processes II and III will be efficient if $\widetilde{D}(\vec{q}, \Omega) \zeta \ll 1$. In the paraxial and monochromatic approximation, the longitudinal wave number $k_{\sigma}^{z}(\vec{q}, \Omega)$ can be expressed as

$$
k_{\sigma}^{z}(\vec{q}, \Omega)=k_{\sigma}+\left[\frac{\omega_{\sigma} n_{\sigma}^{\prime}}{c}+\frac{k_{\sigma}}{\omega_{\sigma}}\right] \Omega+\frac{1}{2} k_{\sigma}^{\prime \prime} \Omega^{2}-\frac{q^{2}}{2 k_{\sigma}}
$$

with $k_{\sigma}^{\prime \prime}=\partial^{2} k / \partial \omega^{2}$ and $n_{\sigma}^{\prime}=\partial n / \partial \omega$ for $\omega=\omega_{\sigma}, \sigma=F, S$. Using this expansion, we can show that $\widetilde{\Delta}(\vec{q}, \Omega)$ and $\widetilde{D}(\vec{q}, \Omega)$ can be written in terms of a common typical spatial frequency $q_{2}=\sqrt{k_{F} / z_{0}}$ and two distinct temporal frequencies $\Omega_{1}=\left[(\omega / c)\left(2 n_{S}^{\prime}-n_{F}^{\prime}\right) z_{0}\right]^{-1}$ and $\Omega_{2}$ $=\left(\left|k_{F}^{\prime \prime}\right| z_{0}\right)^{-1 / 2}$, 


$$
\begin{gathered}
\widetilde{\Delta}(\vec{q}, \Omega)=\Delta s+\operatorname{sgn}\left(k_{F}^{\prime \prime}\right) \frac{\Omega^{2}}{\Omega_{2}^{2}}-\frac{q^{2}}{q_{2}^{2}}, \\
\widetilde{D}(\vec{q}, \Omega)=\Delta s-\frac{\Omega}{\Omega_{1}}-\frac{1}{4}\left(1-\frac{\Delta k}{2 k_{F}}\right) \frac{q^{2}}{q_{2}^{2}} .
\end{gathered}
$$

$\Omega_{2}$ and $q_{2}$ are precisely the scales that are relevant for the OPA in the undepleted pump approximation and set the bandwidths for amplification and squeezing in the fundamental field in terms of temporal and spatial frequency [2]. $\Omega_{1}$ comes in through the first-order dependence of the phase mismatch function $\widetilde{D}(\vec{q}, \Omega)$ on $\Omega$, and determines, as can be seen from Eqs. (29) and (30), the bandwidth for the coupling between the second-harmonic and the fundamental field. Since this coupling is responsible for squeezing generation in the second-harmonic field, as pointed out in [21], $\Omega_{1}$ coincides with the bandwidth for intensity squeezing at $2 \omega$. It is interesting to recall that in the practical case of a $\mathrm{KNbO}_{3}$ crystal considered in [21], the two temporal frequency scales $\Omega_{1}$ and $\Omega_{2}$ were found to be separated by two orders of magnitude: $\Omega_{2} / \Omega_{1} \sim 400$. Different regimes can be hence distinguished according to the value of $\Omega$. For $\Omega \ll \Omega_{1}$, propagation through the nonlinear medium generates a coupling between fundamental and second-harmonic field through all processes represented in Fig. 2. If $\Omega$ is increased to a value such that $\Omega_{1} \ll \Omega \ll \Omega_{2}$, processes II and III become inefficient and Eqs. (29) and (30) simplify to

$$
\frac{\partial}{\partial \zeta} \hat{a}_{F}(\zeta, \vec{q}, \Omega)=-\tilde{c}_{S}(\zeta) \hat{a}_{F}^{\dagger}(\zeta,-\vec{q},-\Omega) \exp [-i \widetilde{\Delta}(\vec{q}, \Omega) \zeta]
$$

$$
\frac{\partial}{\partial \zeta} \hat{a}_{S}(\zeta, \vec{q}, \Omega)=0
$$

Equation (36) describes the parametric amplification of a signal at fundamental frequency through down-conversion of a second-harmonic pump field, the strength of which depends on the position on the longitudinal axis, whereas Eq. (37) indicates that a signal at second-harmonic frequency would cross the nonlinear medium without modification. With respect to spatial frequencies, the scales for the frequencychanging and the parametric down-conversion processes are of the same order of magnitude, as can be seen from Eqs. (34) and (35), so that one does not expect to be able to identify a $q$-value domain in which one particular process will be clearly dominant.

The solution of Eqs. (29) and (30) can be expressed in terms of an input-output transformation for the system of the two fields,

$$
\begin{aligned}
\hat{a}_{F}(\zeta, \vec{q}, \Omega)= & W_{11}(\zeta, \vec{q}, \Omega) \hat{a}_{F}(0, \vec{q}, \Omega)+W_{12}(\zeta, \vec{q}, \Omega) \\
& \times \hat{a}_{F}^{\dagger}(0,-\vec{q},-\Omega)+W_{13}(\zeta, \vec{q}, \Omega) \hat{a}_{S}(0, \vec{q}, \Omega) \\
& +W_{14}(\zeta, \vec{q}, \Omega) \hat{a}_{S}^{\dagger}(0,-\vec{q},-\Omega) \\
\hat{a}_{S}(\zeta, \vec{q}, \Omega)= & W_{31}(\zeta, \vec{q}, \Omega) \hat{a}_{F}(0, \vec{q}, \Omega)+W_{32}(\zeta, \vec{q}, \Omega) \\
& \times \hat{a}_{F}^{\dagger}(0,-\vec{q},-\Omega),+W_{33}(\zeta, \vec{q}, \Omega) \hat{a}_{S}(0, \vec{q}, \Omega) \\
& +W_{34}(\zeta, \vec{q}, \Omega) \hat{a}_{S}^{\dagger}(0,-\vec{q},-\Omega)
\end{aligned}
$$

which connects the field operators at the exit plane of the crystal with those at the input plane. The coefficients $W_{i j}(\zeta, \vec{q}, \Omega)$ are the elements of a $4 \times 4$ matrix $W(\zeta, \vec{q}, \Omega)$ defined as the solution of the following first-order differential equation:

$$
\frac{\partial}{\partial \zeta} W(\zeta, \vec{q}, \Omega)=-A(\zeta, \vec{q}, \Omega) W(\zeta, \vec{q}, \Omega)
$$

with

$A(\zeta, \vec{q}, \Omega)$

$$
=\left(\begin{array}{cc}
0 & \tilde{c}_{S}(\zeta) \exp [-i \widetilde{\Delta}(\vec{q}, \Omega) \zeta] \\
\tilde{c}_{S}^{*}(\zeta) \exp [i \widetilde{\Delta}(-\vec{q},-\Omega) \zeta] & 0 \\
-\sqrt{2} \tilde{c}_{F}(\zeta) e^{i \tilde{D}(\vec{q}, \Omega) \zeta} & 0 \\
0 & -\sqrt{2} \tilde{c}_{F}^{*}(\zeta) \exp [-i \widetilde{D}(-\vec{q},-\Omega) \zeta]
\end{array}\right.
$$

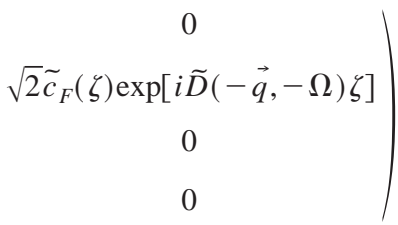

and the initial condition $W(\zeta=0, \vec{q}, \Omega)=I, I$ being the unit matrix. In combination with Eqs. (40) and (41), Eqs. (38) and (39) are the central result of this section and generalize for the case of two coupled fields the multimode squeezing transformation derived in [2] for an OPA in the undepleted pump approximation. Analytical expressions for the coefficients of the input-output transformations (38) and (39) could be derived in [21] for $q=0$ and $\Omega=0$. In the general case, however, no analytical solution is known, and the results presented in the following section were obtained by means of a numerical integration of Eq. (40).

\section{QUANTUM IMAGE PROCESSING}

Following the general ideas of [6,7], we now consider the optical device represented in Fig. 3: the $\chi^{(2)}$-nonlinear crys- 


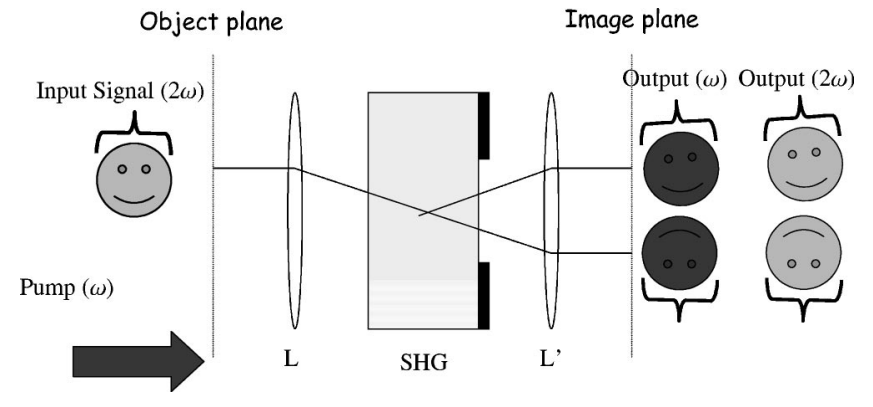

FIG. 3. Scheme of an optical device based on second-harmonic generation. The nonlinear crystal, pumped at frequency $\omega$, is enclosed in a two-lens telescopic system. In the output plane of the crystal, a pupil of finite width represents the finite spatial bandwidth of the system.

tal pumped at frequency $\omega$ investigated in the first part of this paper is enclosed in a two-lens telescopic system. Its role is to map the Fourier plane onto the physical plane, so that this optical system operates on a portion of the input plane rather than on a band of wave vectors [30]. In terms of image processing, this optical device is expected to deliver, for a given input image at frequency $2 \omega$, a pair of symmetric amplified versions of the input image at both fundamental and second-harmonic frequency.

The input-output transformation for the real-space operators can easily be derived taking into account the inputoutput transformation for a lens with focal length $f$ [30], which relates the field operators in the image plane to those in the object plane of the lens,

$$
a_{\text {out }}(\vec{\rho}, t)=\frac{1}{\lambda f} \int d^{2} \rho^{\prime} \exp \left[-i \frac{2 \pi}{\lambda f} \vec{\rho} \cdot \vec{\rho}^{\prime}\right] a_{i n}\left(\vec{\rho}^{\prime}, t\right)
$$

$\lambda$ is the wavelength of the light considered. Since the effects of the telescopic system on the input-output transformation leads to the replacement $\vec{q} \leftrightarrow-\left(2 \pi / \lambda_{\sigma} f\right) \vec{x}$, where $\lambda_{\sigma}$ is the wavelength of the output field considered, we will discuss the results in terms of plane waves with given wave vectors, and remember that the telescopic system converts these wave vectors into positions in the transverse plane. Furthermore, we will assume the temporal evolution of the input image to be slow and put $\Omega \rightarrow 0$ for the calculation of the output images.

The nonlinear crystal is pumped at frequency $\omega$. Considering a vanishing collinear phase mismatch $\Delta k=0$, the amplitudes of the two strong coherent waves at frequency $\omega$ and $2 \omega$ generated in the crystal by the pump, which are needed as an ingredient of the propagation equations for the quantum field operators, are given by Eqs. (27) and (28). The input signal at frequency $2 \omega$ is described by a coherent state $\left|\alpha_{i n}\right\rangle$ characterized by a complex amplitude $\alpha_{i n}(\vec{q}, \Omega)$. With respect to the frequency $\omega,\left|\alpha_{i n}\right\rangle$ is assumed to be in the vacuum state. Hence, we have

$$
\begin{gathered}
\hat{a}_{S}(0, \vec{q}, \Omega)\left|\alpha_{i n}\right\rangle=\alpha_{i n}(\vec{q}, \Omega)\left|\alpha_{i n}\right\rangle, \\
\hat{a}_{F}(0, \vec{q}, \Omega)\left|\alpha_{i n}\right\rangle=0 .
\end{gathered}
$$

As will become clear in the following, the symmetry properties of $\alpha_{i n}(\vec{q}, \Omega)$, exactly as in the OPA case [4], determine the operating regime of the SHG-based optical device: one distinguishes between the phase-insensitive regime, which corresponds to an input signal confined to one half of the object plane of the optical device [8] (an object confined to the upper part of the object plane, for instance, will be characterized by $\alpha_{i n}(\vec{q}, \Omega)=0$ for $\left.q_{y}<0\right)$, and the phasesensitive regime, which refers to the behavior of the optical system in the case of a symmetric input signal, i.e., when $\alpha_{i n}(\vec{q}, \Omega)=\alpha_{i n}(-\vec{q},-\Omega)[7]$.

\section{A. Phase-insensitive configuration}

We start considering the phase-insensitive configuration because it provides a clear picture of the elementary processes, which are responsible for image generation in the SHG-based optical device. Using the input-output transformations (38) and (39), the intensity of an outgoing wave with frequencies $(\vec{q}, \Omega)$ for the input defined by Eq. (43) can easily be calculated. At the fundamental frequency, one finds

$$
\begin{aligned}
\left\langle\hat{a}_{F}^{\dagger}(\zeta, \vec{q}, \Omega) \hat{a}_{F}(\zeta, \vec{q}, \Omega)\right\rangle \\
=(2 \pi)^{3} \delta^{(3)}(0)\left|W_{12}(\zeta, \vec{q}, \Omega)\right|^{2}+(2 \pi)^{3} \delta^{(3)}(0) \\
\quad \times\left|W_{14}(\zeta, \vec{q}, \Omega)\right|^{2}+\left|W_{13}(\zeta, \vec{q}, \Omega)\right|^{2}\left|\alpha_{i n}(\vec{q}, \Omega)\right|^{2} \\
\quad+\left|W_{14}(\zeta, \vec{q}, \Omega)\right|^{2}\left|\alpha_{i n}(-\vec{q},-\Omega)\right|^{2},
\end{aligned}
$$

which indeed does not depend on the phase of the input signal. Four different contributions can be distinguished: the two first terms on the rhs of Eq. (44) are independent of the strength of the input wave and reflect the spontaneous parametric fluorescence, which takes place in the crystal even in the absence of any coherent input signal. The two other contributions are proportional to the intensity of the input wave at $(\vec{q}, \Omega)$ and $(-\vec{q},-\Omega)$, respectively. But because of the particular injection scheme considered here, for a given wave vector $\vec{q}$ at which the output is considered, only one of these two terms is nonvanishing. To fix the ideas, we consider an object confined to the upper part of the object plane. If $q_{y}$ $>0, \quad\left|\alpha_{i n}(-\vec{q},-\Omega)\right|^{2}=0$ and $\left|W_{13}(\zeta, \vec{q}, \Omega)\right|^{2}\left|\alpha_{i n}(\vec{q}, \Omega)\right|^{2}$ describes the intensity distribution of an amplified version of the input image. If $q_{y}<0$, only $\left|W_{14}(\zeta, \vec{q}, \Omega)\right|^{2} \mid \alpha_{i n}(-\vec{q}$, $-\Omega)\left.\right|^{2}$ is nonvanishing and corresponds to a reversed amplified version of the input image. The underlying mechanism is process I of Fig. 2, according to which, second-harmonic photons generated by the strong pump wave inside the crystal decay into pairs of fundamental twin photons propagating in opposite directions [8]. At second-harmonic frequency, we find a similar structure of the output intensity, 


$$
\begin{aligned}
\left\langle\hat{a}_{S}^{\dagger}(\zeta, \vec{q}, \Omega) \hat{a}_{S}(\zeta, \vec{q}, \Omega)\right\rangle & \\
= & (2 \pi)^{3} \delta^{(3)}(0)\left|W_{32}(\zeta, \vec{q}, \Omega)\right|^{2}+(2 \pi)^{3} \delta^{(3)}(0) \\
& \times\left|W_{34}(\zeta, \vec{q}, \Omega)\right|^{2} \\
& +\left|W_{33}(\zeta, \vec{q}, \Omega)\right|^{2}\left|\alpha_{i n}(\vec{q}, \Omega)\right|^{2} \\
& +\left|W_{34}(\zeta, \vec{q}, \Omega)\right|^{2}\left|\alpha_{i n}(-\vec{q},-\Omega)\right|^{2}
\end{aligned}
$$

Therefore, the output at $2 \omega$ is expected to display both an amplified and a phase-conjugate-amplified version of the input image. But the underlying mechanism is different, since there is no three-wave process in the system under consideration, in which two second-harmonic waves with opposite wave vectors and frequency offsets are created simultaneously. As will become clear in the following, these images are generated by the frequency-converting process III acting on the image pair at fundamental frequency, which we have discussed above.

To quantify the efficiency of these mechanisms, we can define, for each of the four output images, a local phaseinsensitive gain as the ratio of the intensity of the output wave under consideration to the intensity of the corresponding input wave, which is given by $\left|\alpha_{i n}(\vec{q}, \Omega)\right|^{2}$. If the contributions to the output intensities due to spontaneous processes can be neglected-this is a reasonable approximation, as has been shown in [6], if a pupil of finite aperture and properly chosen dimensions is located in the output plane of the crystal-the gains can be expressed in terms of the elements of the matrix $W(\zeta, \vec{q}, \Omega)$,

$$
\begin{aligned}
G_{F}(\zeta,+\vec{q},+\Omega) & =\frac{\left\langle\hat{a}_{F}^{\dagger}(\zeta,+\vec{q},+\Omega) \hat{a}_{F}(\zeta,+\vec{q},+\Omega)\right\rangle}{\left\langle\hat{a}_{S}^{\dagger}(0,+\vec{q},+\Omega) \hat{a}_{S}(0,+\vec{q},+\Omega)\right\rangle} \\
& =\left|W_{13}(\zeta, \vec{q}, \Omega)\right|^{2}, \\
G_{F}(\zeta,-\vec{q},-\Omega)= & \frac{\left\langle\hat{a}_{F}^{\dagger}(\zeta,-\vec{q},-\Omega) \hat{a}_{F}(\zeta,-\vec{q},-\Omega)\right\rangle}{\left\langle\hat{a}_{S}^{\dagger}(0,+\vec{q},+\Omega) \hat{a}_{S}(0,+\vec{q},+\Omega)\right\rangle} \\
= & \left|W_{14}(\zeta, \vec{q}, \Omega)\right|^{2}, \\
G_{S}(\zeta,+\vec{q},+\Omega)= & \frac{\left\langle\hat{a}_{S}^{\dagger}(\zeta,+\vec{q},+\Omega) \hat{a}_{S}(\zeta,+\vec{q}, 0)\right\rangle}{\left\langle\hat{a}_{S}^{\dagger}(0,+\vec{q},+\Omega) \hat{a}_{S}(0,+\vec{q},+\Omega)\right\rangle} \\
= & \left|W_{33}(\zeta, \vec{q}, \Omega)\right|^{2}, \\
= & \left|W_{34}(\zeta, \vec{q}, \Omega)\right|^{2}, \\
G_{S}(\zeta,-\vec{q},-\Omega)= & \frac{\left\langle\hat{a}_{S}^{\dagger}(\zeta,-\vec{q},-\Omega) \hat{a}_{S}(\zeta,-\vec{q},-\Omega)\right\rangle}{\left.\hat{a}_{S}^{\dagger}(0,+\vec{q},+\Omega) \hat{a}_{S}(0,+\vec{q},+\Omega)\right\rangle} \\
&
\end{aligned}
$$
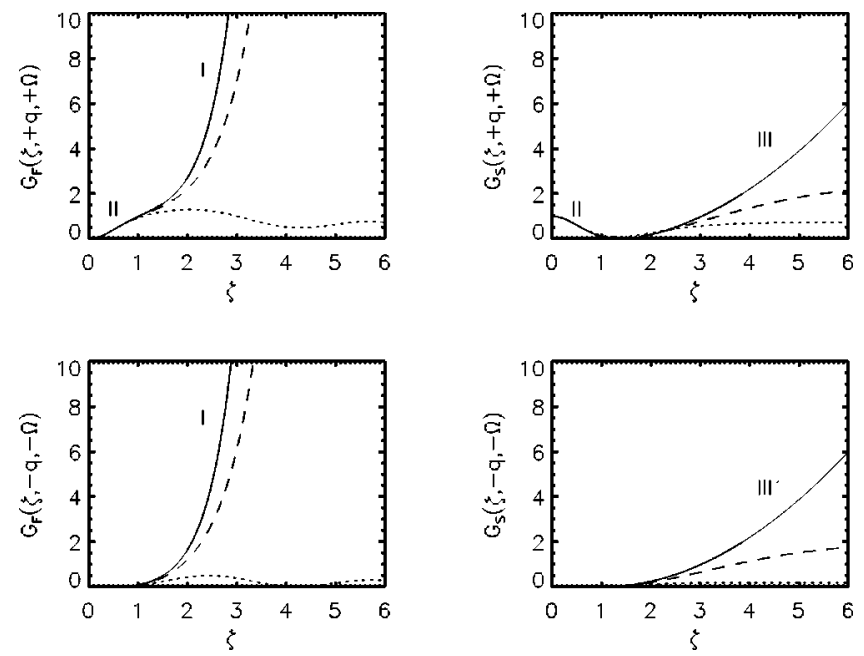

FIG. 4. Phase-insensitive gains as a function of the interaction length $\zeta$ for waves with $\Omega=0$ and different wave numbers: $q$ $=0.5$ (solid line), $q=1.2$ (dashed line), and $q=1.6$ (dotted line) (in units of $q_{2}=\sqrt{k_{F} / z_{0}}$ ). The symbols I, II, III refer to the corresponding dominant elementary processes of Fig. 2. Process III' is the same as process III but with $\vec{q}$ replaced by $-\vec{q}$.

where $\langle\cdots\rangle$ stands for the expectation value in the input coherent state $\left|\alpha_{i n}\right\rangle$ defined through Eqs. (43).

To get some insight into the details of the elementary processes generating the four output images, it is very helpful to first plot the phase-insensitive gains as functions of the interaction length inside the crystal (Fig. 4). At the input plane, we have $G_{F}(\zeta=0,+\vec{q},+\Omega)=G_{F}(\zeta=0,-\vec{q},-\Omega)$ $=G_{S}(\zeta=0,-\vec{q},-\Omega)=0$ and $G_{S}(\zeta=0,+\vec{q},+\Omega)=1$, which simply identifies the input chosen. Increasing the interaction length, a reduction of $G_{S}(\zeta,+\vec{q},+\Omega)$ is first observed, with a simultaneous increase of $G_{F}(\zeta,+\vec{q},+\Omega)$, whereas $G_{F}(\zeta$, $-\vec{q},-\Omega)=G_{S}(\zeta,-\vec{q},-\Omega)$ are still close to zero. This confirms that the dominant process at small interaction lengths is process II of Fig. 2. At $\zeta \simeq 1.4$, the totality of the injected second-harmonic signal turns out to be down-converted. Increasing further the interaction length, a second step in the signal processing is initiated, which, for small wave numbers (solid lines in Fig. 4), is characterized by a rapid and symmetric growth of both fundamental gains at $+q$ and $-q$. Obviously, this is the manifestation of the photon downconversion process labeled $\mathrm{I}$ in Fig. 2. As a consistency check, one observes that at a propagation length $\zeta>1$, already more than $58 \%$ of the pump field has been converted into second-harmonic field, as revealed by Eqs. (27) and (28). Therefore, the interaction lengths for which we observe this rapid growth of both fundamental gains indeed correspond to region II of the crystal (Fig. 1), in which the dominant homogeneous field is the second-harmonic field generated by SHG. As a consequence, this region of the crystal acts principally as an OPA with a z-dependent pump. However, the presence of a weak residual pump field at frequency $\omega$ makes possible a partial frequency up-conversion of the amplified waves at fundamental frequency through processes 

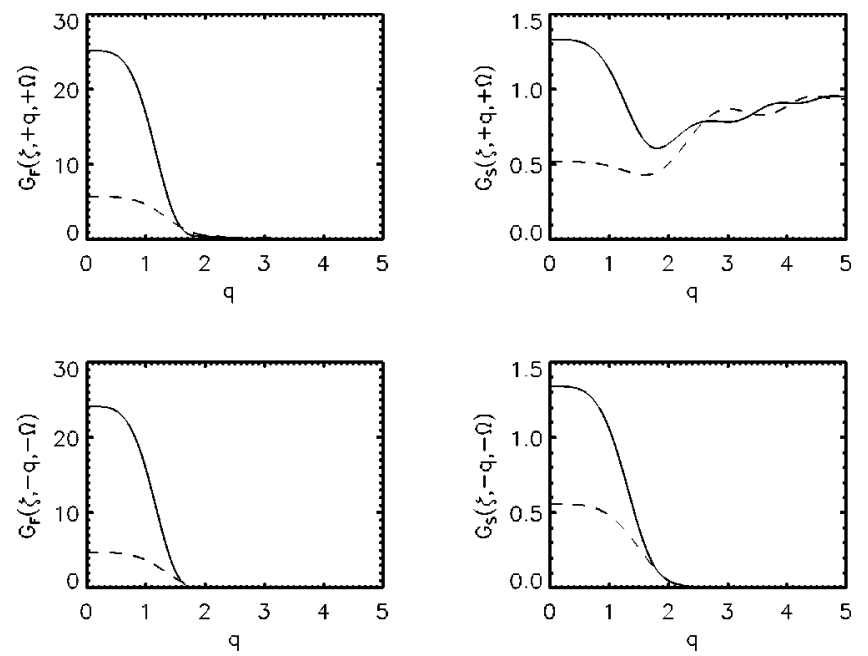

FIG. 5. Phase insensitive gains as a function of the transverse wave number for two different values of the interaction length $\zeta$ $=2.5$ (dashed line) (which is an arbitrary length $>1.4$ ) and $\zeta$ $=3.32$ (solid line) [for which $\left.G_{F}(\zeta,+\vec{q},+\Omega)\right|_{q=\Omega=0} \simeq 25$ ].

III of Fig. 2 and III', which is the process III but involving waves with the transverse wave vector $-\vec{q}$ instead of $\vec{q}$. This mechanism translates into a slow increase of both secondharmonic gains at $+\vec{q}$ and $-\vec{q}$ with increasing interaction length. It is responsible for the formation of the two phaseconjugate output images at the second-harmonic frequency we have already mentioned.

Due to diffraction, which is encoded in the dependence of the phase mismatch functions $\widetilde{D}(\vec{q}, \Omega)$ and $\widetilde{\Delta}(\vec{q}, \Omega)$ on $\vec{q}$ and $\Omega$, the gains will also depend on the spatial and temporal frequency. Considering first the fundamental gains, it turns out that the general consequences of increasing the input wave number can be predicted from the OPA theory: increasing the transverse wave number first translates into a weaker amplification rate of an input signal, and above a critical value $q_{c}=\sqrt{2}$ in the dimensionless units used here, a total suppression of the amplification mechanism is observed and the input signal, instead of being exponentially amplified, will present, as a function of the interaction length, an oscillatory behavior [31]. These predictions coincide with the results of our numerical calculations shown in Fig. 4. For the two second-harmonic gains, we find that increasing the wave number of the input signal leads to a saturation of the output wave intensity, which has a finite asymptotic value in the limit of large propagation lengths.

As far as the optical device sketched in Fig. 3 is concerned, this reduction of the gains at higher transverse wave number will translate into a finite bandwidth for image processing. As for the OPA in the perfect phase-matched case [8], only a finite disk-shaped portion of the input image centered on the beam axis will be efficiently processed by the optical device. This is precisely illustrated in Fig. 5, which shows, for a crystal of a given length, the dependence of the gains on the transverse wave number. For wave numbers $q$ $<1$, all gains have constant, almost $q$-independent values. In real space, this value corresponds to a spatial bandwidth of the optical device that can be estimated to $\rho_{0} \simeq f / \sqrt{k_{F} z_{0}}$. At large transverse wave numbers, say $q>2$, an input wave is seen to basically cross the optical device without being modified: the nonlinear crystal is transparent. However, some structure can be observed in the tail of $G_{S}(\zeta,+\vec{q},+\Omega)$ at large $q$. Closer inspection reveals that it has its counterpart in the tail of $G_{F}(\zeta,+\vec{q},+\Omega)$, which however cannot be seen directly from the graphs displayed in Fig. 5 due to of the different scales used to plot fundamental and secondharmonic gains. This result shows that in the limit of large wave numbers, the dominant process is a weak frequency down-conversion of the input wave, which is consistent with the fact that the variation scale of $\widetilde{D}(\vec{q}, \Omega)$ with respect to the spatial frequency is larger than the one of $\widetilde{\Delta}(\vec{q}, \Omega)$ by a factor 2 .

\section{B. Phase-sensitive configuration}

It is also possible to operate the SHG-based optical device in the so-called phase-sensitive configuration, the difference to the previous scheme being the symmetry of the input signal imposed by the condition $\alpha_{i n}(-\vec{q},-\Omega)=\alpha_{i n}(\vec{q}, \Omega)$. For static signals, this translates into a symmetry of the input image with respect to the beam axis. In the case of the OPA, it is well known that with this symmetry of injection, the output is the result of the coherent superposition of both twin waves produced in an elementary down-conversion process [32]. As a consequence, the amplification is phase sensitive, and this is precisely one requisite for the possibility of amplifying an optical image without deteriorating its signal-tonoise ratio $[7,33]$. It is, therefore, interesting to investigate the optical system considered here with respect to similar properties.

But, before studying the noise behavior of SHG-based signal processing, we first have to investigate the output field distribution for this injection scheme. We begin by noting that the input image being symmetric with respect to the beam axis, the output displays, at each frequency, an image with the same symmetry. Under the same assumptions as in the phase-insensitive case, the ratio of the intensity in a given portion of each output image to the one in the corresponding part of the input image defines the phase-sensitive gains

$$
\begin{aligned}
& G_{F}^{\left(\phi_{i n}\right)}(\zeta, \vec{q}, \Omega)=\mid W_{13}(\zeta, \vec{q}, \Omega) e^{i \phi_{i n}}+W_{14}(\zeta, \vec{q}, \Omega) e^{-\left.i \phi_{i n}\right|^{2}} \\
& G_{S}^{\left(\phi_{i n}\right)}(\zeta, \vec{q}, \Omega)=\left|W_{33}(\zeta, \vec{q}, \Omega) e^{i \phi_{i n}}+W_{34}(\zeta, \vec{q}, \Omega) e^{-i \phi_{i n}}\right|^{2},
\end{aligned}
$$

which both depend on the phase of the input signal $\phi_{\text {in }}$. For simplicity, we only consider input images with an homogeneous phase $\alpha_{i n}(\vec{q}, \Omega)=\left|\alpha_{i n}(\vec{q}, \Omega)\right| e^{i \phi_{i n}}$

This phase sensitivity is illustrated in Fig. 6 for different transverse wave numbers, i.e., for different regions of the transverse plane. On the longitudinal axis $(\vec{q}=0)$, both gains reach maximal values for $\phi_{i n}=\pi / 2+n \pi$, and go through minima at $\phi_{i n}=n \pi$. To understand this observation, it is useful to recall that in a perfect phase-matched OPA, the 

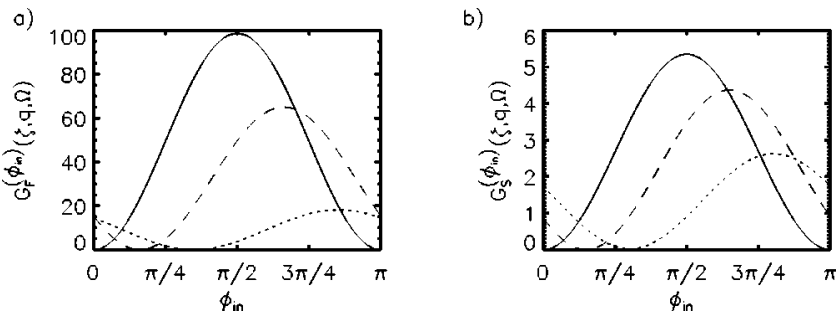

FIG. 6. Phase-sensitive gains for fundamental (a) and secondharmonic (b) fields as a function of the phase of the input signal (in radians) for different values of the transverse wave number $q=0$ (solid line) $q=1$ (dashed line) and $q=1.4$ (dotted line). Interaction length $\zeta=3.32$.

amplification of an input signal (at frequency $\omega$ ) with a phase $\phi_{F, \text { in }}$ is maximal, if $\phi_{S}^{(0)}-2 \phi_{F, \text { in }}=\pi, \phi_{S}^{(0)}$ being the phase of the second-harmonic pump field illuminating the OPA $[2,18]$. If $\phi_{S}^{(0)}-2 \phi_{F, \text { in }}=0$, the input signal turns out to be maximally deamplified. This rule should be valid here as well, provided that $\phi_{F, \text { in }}$ is identified with the phase of the down-converted version of the input signal. In general, $\phi_{F, \text { in }}$ is not identical with the phase $\phi_{\text {in }}$ characterizing the input signal. Inspecting Eqs. (29) and (30), one can show that for $\widetilde{\Delta}(\vec{q}, \Omega)=\widetilde{D}(\vec{q}, \Omega)=0$ - what is fulfilled along the longitudinal axis in the case of perfect phase matching-these two phases are connected in a simple way: $\phi_{F, \text { in }}=\phi_{i n}-\pi$ $-\phi_{F}^{(0)}$, where $\phi_{F}^{(0)}$ is the phase of the pump field illuminating the crystal. Putting these two phase relations together, one predicts that the fundamental gain should have an extremum for $\phi_{i n}=2 \phi_{F}^{(0)}+n \pi / 2$, the maxima corresponding to odd values of $n$. This is exactly what is observed in Fig. 6(a) (in all calculations, the phase of the fundamental pump $\phi_{F}^{(0)}$ was taken equal to 0). Looking at Fig. 6(b), one observes that the second-harmonic gain shows the same behavior. This is not surprising, since image formation at $2 \omega$ can be viewed as the result of a secondary process acting on the fundamental wave propagating through the medium and experiencing either amplification or deamplification. It is hence plausible that the second-harmonic gain will be maximal for those values of the input phase that guarantee a maximal fundamental gain.

Considering off-axis regions of the transverse plane, one observes a shift of the input phase ensuring maximal and minimal gains (dashed and dotted lines in Fig. 6). The main consequence of this observation will be that for an input image with an homogeneous phase, the maximal gain condition can only be satisfied at one point of the transverse plane. However, it should be possible to compensate this positiondependent phase shift through a displacement of the nonlinear crystal with respect to the lenses. This operation, which amounts to superposing a parabolic phase profile to the overall phase of the input image, was shown to achieve, for the OPA, a substantial optimization of the performances of the amplifier [7]. Without elaborating on these considerations, we show in Fig. 7 the phase-sensitive gains as a function of the transverse wave number, for a phase of the input signal chosen so as to have maximal gains at $\vec{q}=0$. The conclusions that can be drawn from these curves are identical with those
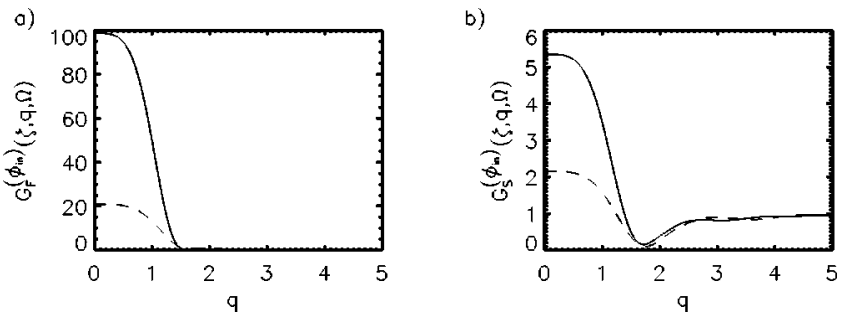

FIG. 7. Phase-sensitive gains for fundamental (a) and secondharmonic (b) fields as a function of the transverse wave number for two different values of the interaction length $\zeta=2.5$ (dashed line) and $\zeta=3.32$ (solid line). The phase of the input image $\left(\phi_{\text {in }}\right.$ $=\pi / 2$ ) is chosen for maximal gain at $q=0$.

obtained for the phase-insensitive injection mode: image processing will be efficient within a region of finite width centered on the beam axis, whereas outside this region, the nonlinear crystal will behave as a transparent medium.

Finally, we investigated the noise properties of the optical device proposed here, which are determined by the quantum fluctuations of the output fields. For this purpose, we first define, at each frequency, the following quadrature operator at phase $\phi_{L O}$ :

$$
\begin{aligned}
& x_{\sigma}^{\left(\phi_{L O}\right)}(\zeta, \vec{q}, \Omega)=\frac{1}{2}\left[a_{\sigma}(\zeta, \vec{q}, \Omega) e^{-i \phi_{L O}+a_{\sigma}^{\dagger}(\zeta,-\vec{q},-\Omega)}\right. \\
& \left.\times e^{i \phi_{L O}}\right] \text {, }
\end{aligned}
$$

which involves the field amplitude operator $a_{\sigma}(\zeta, \vec{q}, \Omega)$ related to the propagation-corrected amplitude $\hat{a}_{\sigma}(\zeta, \vec{q}, \Omega)$

$$
a_{\sigma}(\zeta, \vec{q}, \Omega)=\exp \left\{i\left[k_{\sigma}^{z}(\vec{q}, \Omega)-k_{\sigma}\right] z_{0} \zeta\right\} \hat{a}_{\sigma}(\zeta, \vec{q}, \Omega) .
$$

The exponential factor simply restores the phase accumulated during propagation, which had been for technical reasons factorized out in the definition (16). Unlike the quantities considered before, this phase factor is now important, as was illustrated in [2]: it accounts, in particular, for the modifications of the properties of multimode-squeezed light during propagation in free space.

The variance of $x_{\sigma}^{\left(\phi_{L O}\right)}(\zeta, \vec{q}, \Omega)$ defines the spectrum of squeezing $S_{\sigma}^{\left(\phi_{L O}\right)}(\zeta, \vec{q}, \Omega)$ through the following relation:

$$
\begin{aligned}
& \left\langle x_{\sigma}^{\left(\phi_{L O}\right)}(\zeta, \vec{q}, \Omega), x_{\sigma}^{\left(\phi_{L O}\right)}\left(\zeta, \vec{q}^{\prime}, \Omega^{\prime}\right)\right\rangle \\
& \quad=\frac{1}{4} \delta^{(2)}\left(\vec{q}+\vec{q}^{\prime}\right) \delta\left(\Omega+\Omega^{\prime}\right) S_{\sigma}^{\left(\phi_{L O}\right)}(\zeta, \vec{q}, \Omega)
\end{aligned}
$$

with the usual definition of the variance, $\langle f, g\rangle=\langle f g\rangle$ $-\langle f\rangle\langle g\rangle$. In the case of detectors with perfect quantum efficiency, the spectrum of squeezing coincides with the spectral density for photocurrent fluctuations, normalized to the shot noise level, as measured in a homodyne detection scheme. $\phi_{L O}$ represents the phase of the local oscillator used in this detection setup. 

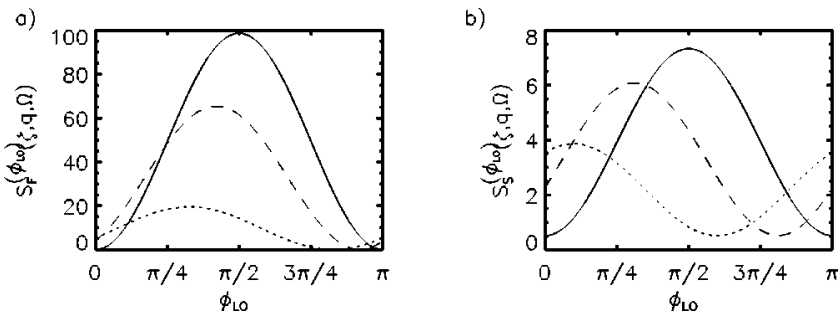

FIG. 8. Spectrum of squeezing $S_{\sigma}^{\left(\phi_{L O}\right)}(\zeta, \vec{q}, \Omega)$ for fundamental (a) and second-harmonic (b) fields as a function of the phase of the local oscillator (in radians) for $\Omega=0$ and different values of the transverse wave number $q=0$ (solid line), $q=1$ (dashed line) and $q=1.4$ (dotted line). Interaction length $\zeta=3.32$.

Figure 8 displays the variation of $S_{\sigma}^{\left(\phi_{L O}\right)}(\zeta, \vec{q}, \Omega)$ as a function of the local oscillator phase. In analogy with a onemode squeezing transformation, changing the local oscillator phase allows one to explore the shape of the uncertainty region covered by the quantum fluctuations of the field. The maximum corresponds to the local oscillator pointing along the quadrature with stretched fluctuations, whereas the minimum indicates the direction of the squeezed quadrature. Considering different values of $\vec{q}$ allows one to observe the effects of diffraction on the squeezing properties of the fields: again, as for the phase-sensitive gains, a shift in the positions of maximum and minimum spectrum of squeezing is observed, which can be interpreted as a rotation of the axis of the uncertainty region. This is a well-known phenomenon in the case of the OPA [2]. Simultaneously, a reduction of the amplitude of oscillations of the spectrum of squeezing indicates a reduction of the squeezing effect with larger $\vec{q}$, with the consequence that the uncertainty region recovers more and more the circular shape characteristic for a coherent state.

These preliminary considerations demonstrate very similar features of the quantum properties of the fields in SHG and in the OPA. In order to be able to appreciate the performances of the SHG device, in particular, with respect to noiseless signal processing, we consider precisely the detection scheme, for which the noiseless amplification was predicted for the OPA [4]: it consists in measuring the sum of the photocurrents from two symmetric pixels in the output plane. Assuming for simplification an ideal quantum efficiency of the detectors, the measured quantity is given by

$$
\begin{aligned}
\left\langle N_{\sigma}(q)\right\rangle= & T_{d}\left(\int_{S_{q}} d^{2} q^{\prime}+\int_{S_{-q}} d^{2} q^{\prime}\right) \\
& \times\left\langle a_{\sigma}^{\dagger}\left(\zeta, \vec{q}^{\prime}, 0\right) a_{\sigma}\left(\zeta, \vec{q}^{\prime}, 0\right)\right\rangle \\
= & 2 T_{d} S_{d} G_{\sigma}^{\left(\phi_{i n}\right)}(\zeta, \vec{q}, 0)\left|\alpha_{i n}(\vec{q}, 0)\right|^{2}
\end{aligned}
$$

in which $S_{q}$ represents the area of the output plane occupied by the photodetector, centered on $q . S_{d}$ represents the pixel area, and $T_{d}$ is the observation time, which is supposed to be large enough, so that the main contributions to the measured current come from the field components with vanishing time frequency offset. The noise affecting this measurement is
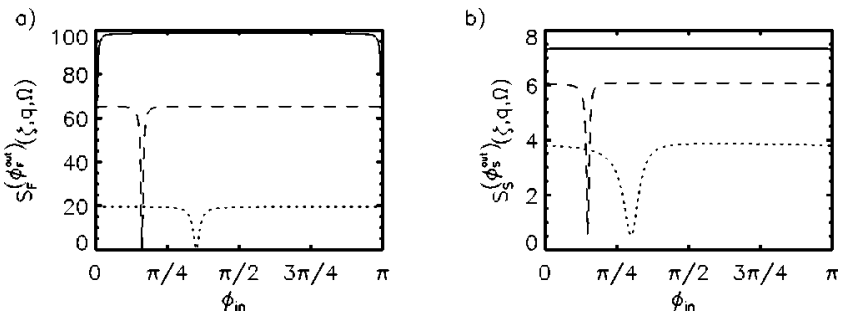

FIG. 9. Intensity fluctuations of fundamental (a) and secondharmonic (b) fields normalized to the shot noise level, as a function of the phase of the input signal for different values of the transverse wave number $q=0$ (solid line) $q=1$ (dashed line) and $q=1.4$ (dotted line). Interaction length $\zeta=3.32$.

characterized by the variance in the mean number of collected photoelectrons $\left\langle\Delta N_{\sigma}^{2}(q)\right\rangle$, which can be calculated. One finds

$$
\left\langle\Delta N_{\sigma}^{2}(q)\right\rangle=S_{\sigma}^{\left(\phi_{L O}\right)}(\zeta, \vec{q}, 0)\left\langle N_{\sigma}(q)\right\rangle,
$$

in which $S_{\sigma}^{\left(\phi_{L O}\right)}(\zeta, \vec{q}, 0)$ can be obtained from the spectrum of squeezing (54), if the local oscillator is taken to coincide with the complex amplitude of the output field. At fundamental frequency, the phase $\phi_{L O}$ has to be taken equal to

$$
\begin{aligned}
\phi_{F}^{\text {out }}= & \arg \left[W_{13}(\zeta, \vec{q}, \Omega) e^{i \phi_{i n}}+W_{14}(\zeta, \vec{q}, \Omega) e^{-i \phi_{i n}}\right] \\
& +\left[k_{F}^{z}(\vec{q}, \Omega)-k_{F}\right] z_{0} \zeta,
\end{aligned}
$$

as can be seen by combining the propagation-corrected output field amplitude at fundamental frequency (50) with the correct propagation phase (53). To compute the intensity fluctuations in the second-harmonic output, $\phi_{L O}$ has to be identified as

$$
\begin{aligned}
\phi_{S}^{\text {out }}= & \arg \left[W_{33}(\zeta, \vec{q}, \Omega) e^{i \phi_{i n}}+W_{34}(\zeta, \vec{q}, \Omega) e^{-i \phi_{i n}}\right] \\
& +\left(k_{S}^{z}(\vec{q}, \Omega)-k_{S}\right) z_{0} \zeta .
\end{aligned}
$$

Figure 9 reveals that the intensity squeezing $S_{\sigma}^{\left(\phi_{L O}\right)}(\zeta, \vec{q}, 0)$, as a function of the input phase of the signal, is almost always given by the maximum value of the spectrum of squeezing, as can be seen from a comparison with Fig. 8, and drops very suddenly to its minimum value when the input phase approaches the value corresponding to the minimal gain. This simply reflects the fact that we are considering parameters for which the maximal gain is high (of the order of 100 for an interaction length $\zeta=3.32$ ), so that the orientation of the output field amplitude in the complex plane is dominated by the "amplified" quadrature. Only for input signal phases close to fulfill the condition for minimal gain, the "deamplified" quadrature comes into play, and the phase of the output field varies rapidly, translating into a rapid change of orientation of the local oscillator. Furthermore, the diffraction-induced shift in the input phase dependence can be clearly seen when looking at Fig. 9 .

The noise performance of an optical device is described by the noise figure 
a)

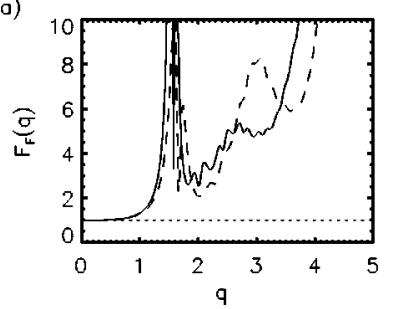

b)

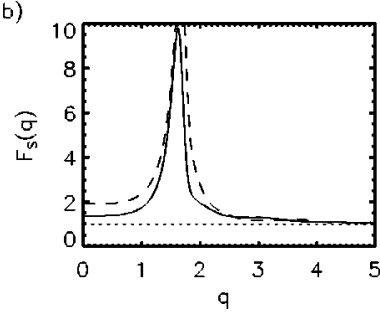

FIG. 10. Noise figure at fundamental (a) and second-harmonic (b) frequency as a function of the transverse wave number for two different values of the interaction length $\zeta=3.32$ (solid line) and $\zeta=2.5$ (dashed line). As a dotted line, the smallest possible value $F_{\sigma}(q)=1$ for a linear system.

$$
F_{\sigma}(q)=\frac{\mathcal{R}^{\text {in }}(q)}{\mathcal{R}_{\sigma}^{\text {out }}(q)}
$$

where $\mathcal{R}^{\text {in }}\left(\mathcal{R}_{\sigma}^{\text {out }}\right.$ ) represents the signal-to-noise ratio in the input image (each output image), and is defined as

$$
\mathcal{R}(q) \equiv \frac{\left\langle N_{\sigma}(q)\right\rangle^{2}}{\left\langle\Delta N_{\sigma}^{2}(q)\right\rangle} .
$$

Collecting the results of Eqs. (55) and (56), one easily observes that the noise figure is given by the ratio of the intensity-squeezing spectrum to the phase-sensitive gain

$$
F_{\sigma}(q)=\frac{S_{\sigma}^{\phi_{\sigma}^{\text {out }}(\zeta, \vec{q}, 0)}}{G_{\sigma}^{\left(\phi_{\text {in }}\right)}(\zeta, \vec{q}, 0)} .
$$

Choosing again the phase of the input signal for maximal phase-sensitive gains at $q=0$, the results of our numerical computation of the noise figure for each output are presented in Fig. 10. Since all this analysis is done in the linearization approach, the propagation equations for the field operators are linear and the noise figure can never be less than 1 [33]. This smallest possible value, which has been plotted for comparison as a dotted line, corresponds to a noiseless operation, in which the noise level is preserved during processing. In the domain of wave numbers, in which image processing is efficient, we find that the fundamental output shows the same level of noise as the input image: the SHGbased device operates without adding noise to the signal. If we consider the output at frequency $2 \omega$, we see that the noise figure is slightly above 1 . With respect to this output frequency, image processing is hence affected by a degradation of the signal-to-noise ratio. However, increasing the interaction length, the figure noise is seen to approach 1 for the spatial frequencies inside the bandwidth for image processing. Finally, one notes that, in the transparency region, the noise figure with respect to the second-harmonic output is equal to 1 , since the input signal is unaffected by the optical system, whereas the noise figure for the fundamental output diverges, as a consequence of a vanishing output intensity at $\omega$ in the limit $|\vec{q}| \rightarrow \infty$. The structure observed in the tail of $F_{F}(q)$ can be traced back to the interplay of the diffractioninduced rotation of the uncertainty region with respect to the

output field amplitude with some structure in the $q$ dependence of the fundamental gain.

\section{CONCLUSIONS AND OUTLOOK}

In this paper, the spatial behavior of light generated by a $\chi^{(2)}$-nonlinear crystal pumped at the fundamental frequency was investigated. Focusing on the problem of the propagation of coherent input signals, we found that the process of second-harmonic generation, which spontaneously takes place in the crystal, opens new possibilities in quantum image processing. Essentially, this can be traced back to the fact that a SHG-based optical device is equivalent to an OPA combined with a frequency converter. This combination allows, for instance, to switch a given optical input image at frequency $2 \omega$ down to frequency $\omega$ before amplifying and cloning it. As a result, the output at fundamental frequency displays two amplified versions of the input image, which are symmetric with respect to the system axis. Investigating the quantum fluctuations in this output, we conclude that for a symmetric input image with a properly chosen phase, this two-step image processing can operate preserving the signalto-noise ratio. This can be a very valuable property when processing very weak input signals. In addition, the frequency converter "part" of the device is also able to convert a small fraction of this output up to frequency $2 \omega$, generating a pair of symmetric images at second-harmonic frequency. The output intensity at $2 \omega$ was found to be much weaker than the fundamental output and affected by some excess noise, which, however, can be lowered by considering larger interaction lengths. More quantitatively, considering one of the numerical examples $\zeta=2.5$ used in this paper, we found in the phase-sensitive configuration a ratio of output intensity at fundamental frequency to input intensity of 20, with an unchanged noise level. The output at $2 \omega$ was found to be roughly twice the input intensity, with a noise level approximately increased by the same factor. These values were found to be valid for a region of finite width $\rho_{0}$ $\simeq f / \sqrt{k_{F} z_{0}}$ centered on the beam axis.

However, these interesting properties of SHG-based signal processing require interaction lengths above a minimal length $\zeta_{\min } \sim 1.4$, which is the interaction length necessary to first down convert the input signal. This value is slightly above the actual experimental possibilities. As a matter of fact, a good second-harmonic conversion rate obtained experimentally is of the order of $66 \%$ [34], corresponding to an interaction length $\zeta_{\text {exp }} \sim 1.1$. Therefore, to be able to observe the effects predicted here, an increase of the available interaction length by $50 \%$ is necessary. It seems reasonable that this will be possible in the near future, either increasing the pump power, or the nonlinearity of the crystal, as reported, for example, in [35], or optimizing further the conversion efficiency [34].

From a general point of view, the work reported here opens interesting perspectives for further investigation. A first objective would aim at a better understanding, on an analytical basis, of the spatial behavior of the fields generated by SHG. In particular, since the two field input-output transformations (38) and (39) are the generalizations to the 
case of two coupled fields at frequency $\omega$ and $2 \omega$ of a multimode squeezing transformation, it would be interesting to be able to interpret it in terms of simple geometrical transformations in the complex plane of the field amplitudes. As for the OPA, this would allow to predict most of the properties of quantum image processing in a very simple way.

Whereas the study presented here was restricted to the case of perfect phase matching, it was shown that SHG with a finite phase mismatch could present interesting properties [20]. In particular, it was found that the second-harmonic field could be highly squeezed, whereas a $50 \%$ squeezing is the maximum available in the perfect phase-matching situation. As far as the OPA is concerned, an imperfect phase matching has the consequence that the region of the transverse plane for which the image processing is optimal is no more a circular spot centered on the beam axis, but turns out to be ring shaped. With a proper choice of the phase mismatch, it is hence possible to optimally process off-axis regions of the transverse plane [8]. Since the SHG device studied here is based on a combination of both processes, it would be interesting to see if, for a finite phase mismatch, one could simultaneously exploit both advantages.

\section{ACKNOWLEDGMENTS}

Financial support from the European Commission Projects QSTRUCT (FMRX-CT96-0077) and QUANTIM (IST-2000-26019), as well as from CONOCE MCyT (Spain) (Project No. BFM2000-1108), is acknowledged. We also acknowledge helpful discussions with M. Kolobov.
[1] Y. Yamamoto, S. Machida, S. Saito, N. Imoto, T. Yanagawa, M. Kitagawa, and G. Bjork, in Progress in Optics, edited by E. Wolf (North-Holland, Amsterdam, 1990), Vol. 28, p. 89.

[2] M.I. Kolobov, Rev. Mod. Phys. 71, 1539 (1999).

[3] L.A. Lugiato, E. Brambilla, and A. Gatti, in Advances in Atomic, Molecular, and Optical Physics, edited by B. Bederson and H. Walther (Academic, Boston, 1999), Vol. 40, p. 229.

[4] A. Gatti, E. Brambilla, L.A. Lugiato, and M.I. Kolobov, J. Opt. B: Quantum Semiclassical Opt. 2, 196 (2000).

[5] M.I. Kolobov and I.V. Sokolov, Zh. Éksp. Theor. Fiz. 96, 1945 (1989) [Sov. Phys. JETP 69, 1097 (1989)]; Phys. Lett. A 140, 101 (1989); Europhys. Lett. 15, 271 (1991).

[6] M.I. Kolobov and L.A. Lugiato, Phys. Rev. A 52, 4930 (1995).

[7] I.V. Sokolov, M.I. Kolobov, and L.A. Lugiato, Phys. Rev. A 60, 2420 (1999).

[8] A. Gatti, E. Brambilla, L.A. Lugiato, and M.I. Kolobov, Phys. Rev. Lett. 83, 1763 (1999); J. Opt. B: Quantum Semiclassical Opt. 1, 1763 (1999).

[9] E. Brambilla, A. Gatti, P. Navez, and L.A. Lugiato, e-print quant-ph/0010108.

[10] C. Fabre, J.B. Fouet, and A. Maitre, Opt. Lett. 25, 76 (2000).

[11] N. Treps, U. Andersen, B. Buchler, P.K. Lam, A. Maitre, H. Bachor, and C. Fabre (unpublished).

[12] I.V. Sokolov, Opt. Spektrosk. 70, 393 (1991).

[13] I.V. Sokolov, J. Opt. B: Quantum Semiclassical Opt. 2, 179 (2000).

[14] M.I. Kolobov and P. Kumar, Opt. Lett. 18, 849 (1993).

[15] M.I. Kolobov and C. Fabre, Phys. Rev. Lett. 85, 3789 (2000).

[16] M.L. Marable, S.K. Choi, and P. Kumar, Opt. Express 2, 84 (1998).

[17] S.K. Choi, M. Vasilyev, and P. Kumar, Phys. Rev. Lett. 83, 1938 (1999).
[18] J.A. Armstrong, N. Bloembergen, J. Ducuing, and P.S. Pershan, Phys. Rev. 127, 1918 (1962).

[19] R.D. Li and P. Kumar, Opt. Lett. 18, 1961 (1993).

[20] R.D. Li and P. Kumar, Phys. Rev. A 49, 2157 (1994).

[21] Z.Y. Ou, Phys. Rev. A 49, 2106 (1994).

[22] A. Andreoni, M. Bondani, G.M. D'Ariano, and M.G.A. Paris, Eur. Phys. J. D 13, 415 (2001).

[23] G.M. D’Ariano, M.G.A. Paris, and M.F. Sacchi, Nuovo Cimento Soc. Ital. Fis., B B114, 339 (1999).

[24] C.L. Tang and L.K. Cheng, Fundamentals of Optical Parametric Processes and Oscillators, Laser Science and Technology Vol. 20 (Harwood Academic, Amsterdam, 1995), pp. 30 and 31.

[25] A. Gatti (private communication).

[26] M.K. Olsen, R.J. Horowicz, L.I. Plimak, N. Treps, and C. Fabre, Phys. Rev. A 61, 021803 (2000).

[27] M.K. Olsen and R.J. Horowicz, Opt. Commun. 168, 135 (1999).

[28] M.K. Olsen, L.I. Plimak, M.J. Collet, and D.F. Walls, Phys. Rev. A 62, 023802 (2000).

[29] M.K. Olsen, K. Dechoum, and L.I. Plimak, Opt. Commun. 190, 261 (2001).

[30] M.I. Kolobov and L.A. Lugiato, Phys. Rev. A 52, 4930 (1995).

[31] A. Berzanskis, W. Chinaglia, L.A. Lugiato, K.-H. Feller, and P. Di Trapani, Phys. Rev. A 60, 1626 (1999).

[32] E. Lantz and F. Devaux, Quantum Semiclassic. Opt. 9, 279 (1997).

[33] C.M. Caves, Phys. Rev. D 26, 1817 (1982).

[34] S. Yu and M. Weiner, J. Opt. Soc. Am. B 16, 1300 (1999).

[35] J. Liu, Z. Wang, S. Zhang, J. Wang, H. Chen, Z. Shao, and M. Kiang, Opt. Commun. 195, 267 (2001). 